\title{
'
}

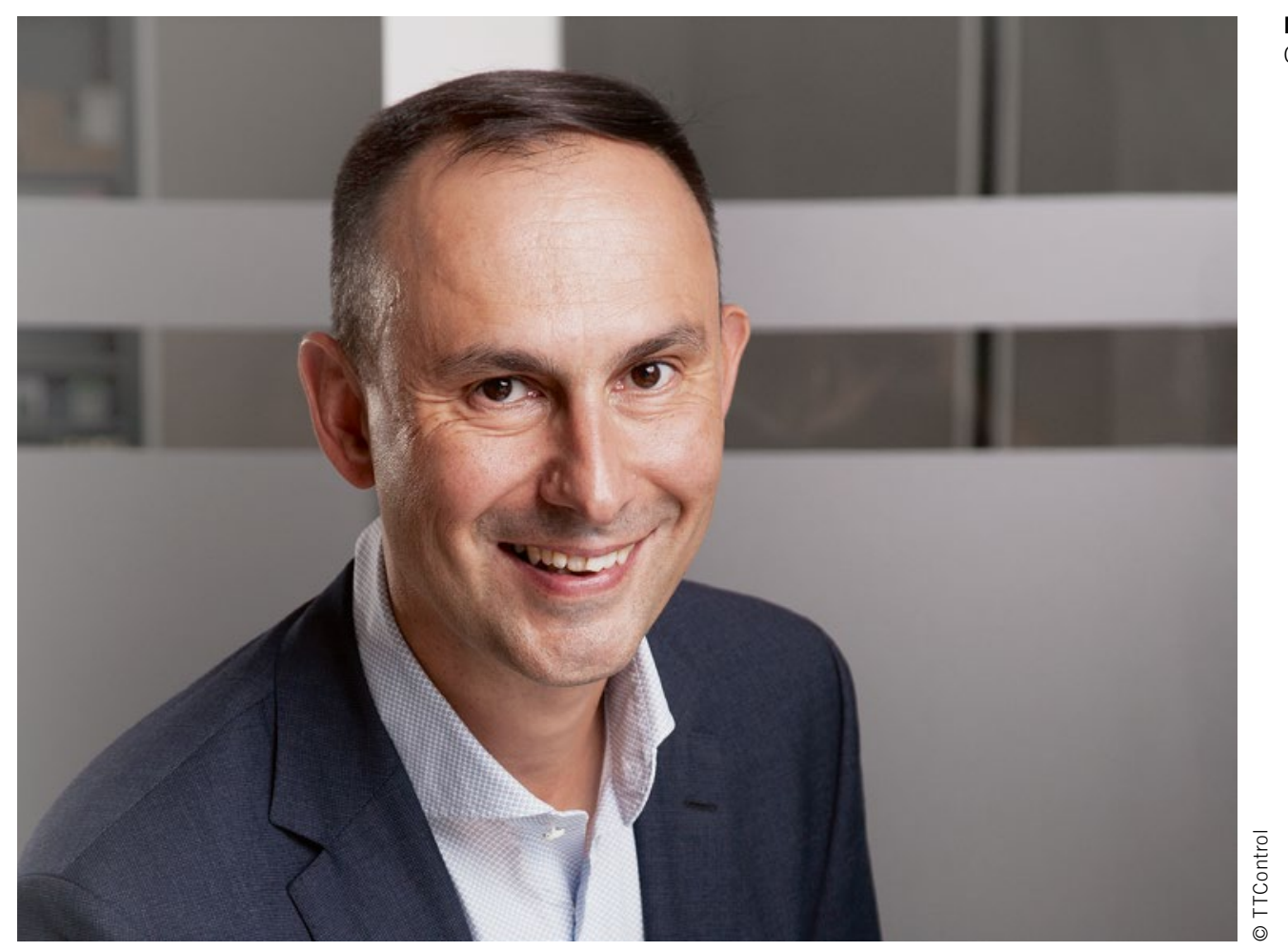

Manfred Prammer

Geschäftsführer TTControl

\section{Zertifizierung spart Kosten}

Viele Hersteller mobiler Maschinen verlieren Marktanteile, weil ihren Produkten aufgrund zu teurer und zeitaufwendiger Zertifizierungsprozesse Sicherheitszertifizierungen fehlen. Es ist zu empfehlen, dass OEMs ihre Strategie in dieser Hinsicht überdenken, denn sicherheitszertifizierte mobile Maschinen gewährleisten nicht nur höchste Sicherheit und Zuverlässigkeit, sondern sind mit gutem Grund häufig in Ausschreibungen vorgegeben. Die Sicherheitszertifizierung reduziert zudem Haftungsrisiken und Regressansprüche gegenüber dem Hersteller im Schadensfall. Ein kostengünstigerer Weg zu einer erfolgreichen Zertifizierung ist hier die Verwendung sicherheitszertifizierter Komponenten.

Beim Thema Sicherheit geht es darum, Risiken auf ein akzeptables Maß zu reduzieren. Um dieses Ziel zu erreichen, verlangen Sicherheitsnormen, dass bei der Entwicklung und Herstellung von Produkten bestimmte komplexe und zeitaufwendige Prozesse eingehalten werden. Im Rahmen dieser Prozesse müssen Sicherheitsrisiken identifiziert und spezifische Anforderungen festgelegt werden, um ihnen entgegenzuwirken. Zur Erfüllung der Anforderungen müssen sicherheitsrelevante Funktionen oder Mechanismen etabliert werden. Diese sind in der Regel einigen Komponenten der Fahrzeugarchitektur zugeordnet, beispielsweise elektronischen Steuergeräten (ECUs). Heutige ECUs sind leistungsstark und können vielfältige Überwachungs- und Diagnosefunktionen ausführen. Tritt ein sicherheitskritischer
Fehler auf, wechselt die ECU in den sicheren Zustand. Genauer gesagt werden sicherheitskritische Ausgänge abgeschaltet.

Die in den ECUs implementierten Diagnose- und Fehlerreaktionsmechanismen sind technisch sehr komplex. Ihre Konzeption und Umsetzung erfordern hochqualifizierte Experten, die sowohl knapp als auch teuer sind. Mit dem Einsatz zertifizierter Komponenten lassen sich Skaleneffekte erzielen und wertvolle, qualifizierte Personalressourcen einsparen oder anderweitig einsetzen, indem häufig auftretende komplexe Probleme von einem spezialisierten Zulieferer einmalig für viele Kunden gelöst werden. Für die OEMs gibt es trotzdem noch genug zu tun. Natürlich können zertifizierte Komponenten wie ECUs nicht garantieren, dass das auf der Plattform implementierte Endkontrollsystem die Normen erfüllt. Da die auf einer ECU laufende OEM-Applikation erst einmal nicht bekannt ist, kann nur von einer angenommenen Sicherheitsfunktion ausgegangen werden. Die Norm ISO 26262 definiert solche Systeme als „Safety Element out of Context“ (SEooC). Da es keine elektronische Komponente gibt, die universell einsetzbar ist, muss jeder OEM weiterhin anhand seiner spezifischen Bedingungen und Bedürfnisse die Vor- und Nachteile abwägen. Generell ist die Verwendung zertifizierter elektronischer Komponenten von Vorteil, da sie den Umfang der Systemzertifizierung reduziert und sich die Markteinführungszeit verkürzt, weil Probleme bereits in einer gebrauchsfertigen Komponente gelöst wurden. 